\title{
Evaluation of Translation Velocity Control by Auxiliary Coils for the Collisional Merging Formation of FRCs by 2-D Resistive MHD Simulation*)
}

\author{
Daichi KOBAYASHI, Tomohiko ASAI, Tsutomu TAKAHASHI, Junichi SEKIGUCHI, \\ Hiroshi GOTA $^{1)}$, Sean DETTRICK ${ }^{1)}$, Yung MOK ${ }^{1)}$, Michl BINDERBAUER ${ }^{1)}$ \\ and Toshiki TAJIMA ${ }^{1,2)}$ \\ College of Science and Technology, Nihon University, Tokyo 101-8308, Japan \\ ${ }^{1)}$ TAE Technologies, Inc., Foothill Ranch, CA 92610, USA \\ ${ }^{2)}$ Department of Physics and Astronomy, University of California at Irvine, Irvine, CA 92697, USA
}

(Received 29 November 2019 / Accepted 20 February 2020)

\begin{abstract}
Previously, the collisional merging formation of FRCs has been developed to generate high performance FRCs. A drastic increase in the excluded flux and total temperature were observed in the FRCs generated with this technique; furthermore, these phenomena strongly depend on the translation velocity of individual plasmoids. However, the dependence of the merged-FRC performance on the translation velocity has not been studied in detail. To study the dependence, the effect of installed auxiliary coils on the formation region on the translation velocity of FRCs in the FAT-CM device has been evaluated by the simulation with a two-dimensional resistive magnetohydrodynamics code, which is called Lamy Ridge. The control of translation velocity by using the auxiliary coils has beenwas demonstrated on the order of several tens of $\mathrm{km} / \mathrm{s}$ in the simulation. The trapped poloidal flux and the total temperature of simulated FRCs have increased depending on the translation velocity.

(C) 2020 The Japan Society of Plasma Science and Nuclear Fusion Research
\end{abstract}

Keywords: magnetically confined plasma, field-reversed configuration, high beta plasma, FRC merging, resistive MHD simulation

DOI: $10.1585 /$ pfr. 15.2402020

\section{Introduction}

A field-reversed configuration (FRC) is a compact torus that has poloidal field and zero or small amount of self-generated toroidal field [1,2]. FRC has an extremely high beta value $(\langle\beta\rangle \sim 1)$ that makes the potential for using aneutronic fusion fuels such as D- $-{ }^{3} \mathrm{He}$ and $\mathrm{p}-{ }^{11} \mathrm{~B}[3]$.

Recently, the technique of collisional merging formation of FRCs has been developed to generate high performance FRCs [4]. Two initial-FRCs formed by the fieldreversed theta pinch (FRTP) method, which is a conventional technique to form an FRC are translated at super Alfvénic velocity toward each other so that they collide and merge into a single FRC state. In the collisional merging process, a drastic increase in the excluded flux and the total temperature of merged-FRCs was observed on the FAT$\mathrm{CM}$ device and the C-2/C-2U device $[4,5]$. These phenomena strongly depend on the translation velocity of individual plasmoids [6]. However, the dependence of the merged-FRC performance, such as the excluded flux and the total temperature, on the translation velocity has not been studied in detail. To study the dependence, a method for controlling the translation velocity of initial-FRCs that

author'se-mail: csda19001@g.nihon-u.ac.jp

*) This article is based on the presentation at the 28th International Toki Conference on Plasma and Fusion Research (ITC28). utilizes the auxiliary coils has been proposed and evaluated by the two-dimensional resistive magnetohydrodynamics (MHD) simulation.

\section{FAT-CM Device}

Figure 1 shows the schematic diagram of the FAT-CM device and the axial profile of the external guide magnetic field. This device consists of a central confinement chamber, quasi-steady state confinement coils, and two FRTP formation sections, called "R-formation" and "Vformation," respectively. Each formation section consists of a transparent quartz tube ( $0.25 \mathrm{~m}$ in ID) and theta-pinch coils. The central confinement chamber is made of stainless steel $(0.78 \mathrm{~m}$ in ID) and serves as a flux conserver during translation, collision, and merging processes because the skin time $(\sim 0.5 \mathrm{~ms})$ is much longer than their timescale. In the typical FAT-CM device operation, theta pinch coils generate the main compression magnetic field of $\sim 0.4 \mathrm{~T}$, and quasi-steady state confinement coils generate the external magnetic field of $\sim 0.06 \mathrm{~T}$. Initial-FRCs, which are generated in each formation, $\sim 0.06 \mathrm{~m}$ radius and $\sim 1 \mathrm{~m}$ length, have $\sim 2.5 \times 10^{21} \mathrm{~m}^{-3}$ of electron density and $\sim 200 \mathrm{eV}$ of total temperature. Initial-FRCs are translated due to the gradient of the external guide magnetic field into the central confinement chamber. The translation veloc- 


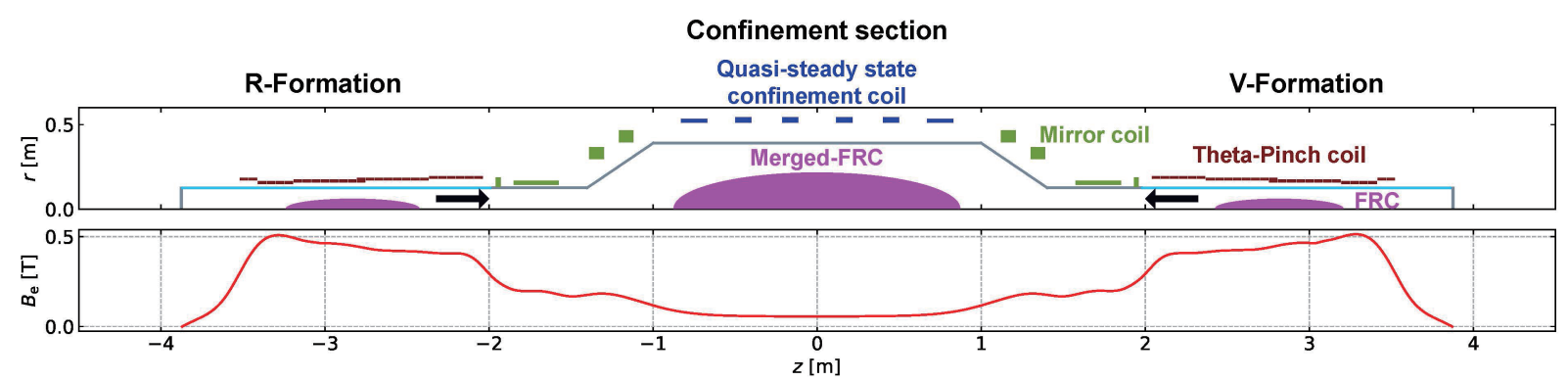

Fig. 1 Schematic diagram of the FAT-CM device and the axial profile of the external guide magnetic field.

ity is $150-200 \mathrm{~km} / \mathrm{s}$, which is faster than the Alfvénic velocity $(\sim 70 \mathrm{~km} / \mathrm{s})$ calculated with the typical electron density of merged-FRCs and the external magnetic field at the midplane of the FAT-CM device $(\sim 0.06 \mathrm{~T})$. Merged-FRCs, $\sim 0.2 \mathrm{~m}$ radius and $\sim 1.6 \mathrm{~m}$ length with $0.5-1.0 \mathrm{mWb}$ of estimated trapped poloidal flux, have $\sim 1.5 \times 10^{20} \mathrm{~m}^{-3}$ of electron density and $100 \mathrm{eV}$ of total temperature.

\section{Model Equations}

Lamy Ridge is a two-dimensional MHD code, which has been developed to simulate the entire process of formation, translation, and collisional merging of FRCs in the C-2 device [7,8]. The simulation of merged-FRCs in the FAT-CM device with the Lamy Ridge code also appeared in a previous study [9]. The code consists of the resistive MHD equations supplemented with an energy equation, and neutral fluid equations. The equations for plasma and neutrals interactively exchange their momentum, density, and energy through ionization. The energy equation incorporates non-adiabatic effects, including thermal conduction, ionization loss, and radiation loss. In the code, plasma is described by

$$
\begin{aligned}
& \frac{\partial n}{\partial t}+\nabla \cdot n \mathbf{u}=n n_{\mathrm{n}}\left\langle\sigma_{\mathrm{ion}} \mathrm{v}\right\rangle, \\
& M n \frac{D \mathbf{u}}{D t}=\mathbf{J} \times \mathbf{B}-\nabla P+\nabla \cdot v \nabla \mathbf{u} \\
& \quad-M n_{\mathrm{n}} v_{\mathrm{ni}}\left(\mathbf{u}-\mathbf{u}_{\mathrm{n}}\right)-n n_{\mathrm{n}}\left\langle\sigma_{\mathrm{ion}} \mathrm{v}\right\rangle \mathbf{u}, \\
& \frac{\partial w}{\partial t}+\nabla \cdot\left(\frac{\gamma P}{\gamma-1}+\frac{M n \mathrm{u}^{2}}{2}\right) \mathbf{u} \\
& =\mathbf{u} \cdot \mathbf{J} \times \mathbf{B}+n k_{\mathrm{B}}\left(\frac{\partial T_{\mathrm{i}}}{\partial t}+\frac{\partial T_{\mathrm{e}}}{\partial t}\right), \\
& n k_{\mathrm{B}} \frac{\partial T_{\mathrm{i}}}{\partial t}=v_{\mathrm{eq}} n k_{\mathrm{B}}\left(T_{\mathrm{e}}-T_{\mathrm{i}}\right)+\nabla \cdot \chi_{\mathrm{i}} \nabla T_{\mathrm{i}}, \\
& n k_{\mathrm{B}} \frac{\partial T_{\mathrm{e}}}{\partial t}=v_{\mathrm{eq}} n k_{\mathrm{B}}\left(T_{\mathrm{i}}-T_{\mathrm{e}}\right)+\nabla \cdot \chi_{\mathrm{e}} \nabla T_{\mathrm{e}} \\
& \quad+\eta J^{2}-R-I .
\end{aligned}
$$

In addition, neutrals are described by

$$
\begin{aligned}
& \frac{\partial n_{\mathrm{n}}}{\partial t}+\nabla \cdot n_{\mathrm{n}} \mathbf{u}_{\mathrm{n}}=-n n_{\mathrm{n}}\left\langle\sigma_{\text {ion }} \mathrm{v}\right\rangle, \\
& M n_{\mathrm{n}} \frac{D \mathbf{u}_{\mathrm{n}}}{D t}=-k_{\mathrm{B}} \nabla\left(T_{\mathrm{n}} n_{\mathrm{n}}\right)+\nabla \cdot v \nabla \mathbf{u}_{\mathrm{n}} \\
& \quad-M n_{\mathrm{n}} v_{\mathrm{ni}}\left(\mathbf{u}_{\mathrm{n}}-\mathbf{u}\right)+n n_{\mathrm{n}}\left\langle\sigma_{\text {ion }} \mathrm{v}\right\rangle \mathbf{u}_{\mathrm{n}},
\end{aligned}
$$

$$
\frac{\partial w_{\mathrm{n}}}{\partial t}+\nabla \cdot\left(\frac{\gamma P_{\mathrm{n}}}{\gamma-1}+\frac{M n_{\mathrm{n}} \mathrm{u}_{\mathrm{n}}^{2}}{2}\right) \mathbf{u}_{\mathrm{n}}=\nabla \cdot \chi_{\mathrm{n}} \nabla T_{\mathrm{n}}
$$

Here, $n$ is the density, $\mathbf{u}$ is the flow velocity, $\left\langle\sigma_{\text {ion }} v\right\rangle$ is the ionization reaction rate, $v$ is the viscosity, $M$ is the ion mass, $\mathbf{J}$ is the current density in the toroidal direction, $\mathbf{B}$ is the magnetic flux density, $P$ is the plasma pressure, $\gamma$ is the specific heat ratio, $T$ is temperature, $v_{\mathrm{eq}}$ is the reciprocal of the energy equilibration time, $\chi$ is the thermal conductivity, and $R$ and $I$ are the radiative energy loss and ionization loss, respectively. Subscripts "i," "e," and " $n$ " indicate ion, electron, and neutrals, respectively. The total of internal and kinetic energy density is defined as

$$
w \equiv \frac{P}{(\gamma-1)}+\frac{M n u^{2}}{2} .
$$

Resistivity includes Spitzer resistivity $\eta_{\mathrm{S}}$, Chodura resistivity, and numerical resistivity by diffusivity $D_{\eta}$,

$$
\eta=\eta_{\mathrm{S}} D_{\mathrm{S}}+\mu_{0} D_{\eta}+C_{\mathrm{C}}\left(1-\exp \left[\frac{-V_{\mathrm{D}}}{f_{\mathrm{C}} V_{\mathrm{T}}}\right]\right) \frac{m_{\mathrm{e}}}{e \sqrt{M n \varepsilon_{0}}}
$$

Here, $\varepsilon_{0}$ and $\mu_{0}$ are the permittivity and magnetic permeability of free space, and $V_{\mathrm{D}}$ and $V_{\mathrm{T}}$ are the drift velocity and ion acoustic velocity, respectively. The viscosity, $D_{\mathrm{S}}$, $C_{\mathrm{C}}, f_{\mathrm{C}}$, and $D_{\eta}$ are adjustable values. In addition, magnetic field is described by

$$
\begin{aligned}
& \mathbf{B}=\nabla \Psi \times \nabla \theta, \\
& \frac{\partial \psi}{\partial t}=r(\mathbf{u}+\mathbf{B})_{\theta}+\frac{\eta}{\mu_{0}} \Delta^{*} \Psi, \\
& \mathbf{J}=-\frac{\Delta^{*} \Psi}{\mu_{0} r} \widehat{\boldsymbol{\theta}},
\end{aligned}
$$

where $\Psi$ is the poloidal flux per radian, and $\widehat{\boldsymbol{\theta}}$ is the unit vector in the toroidal direction. The code advances equations in time by using a semi-implicit algorithm to take large time steps.

The simulation result under the typical condition of FAT-CM experiments is shown in Fig. 2. The entire process of collisional merging formation of FRCs has been simulated, also the performance of simulated mergedFRCs was approximately consistent with that of experimental FRCs (Fig. 3). 


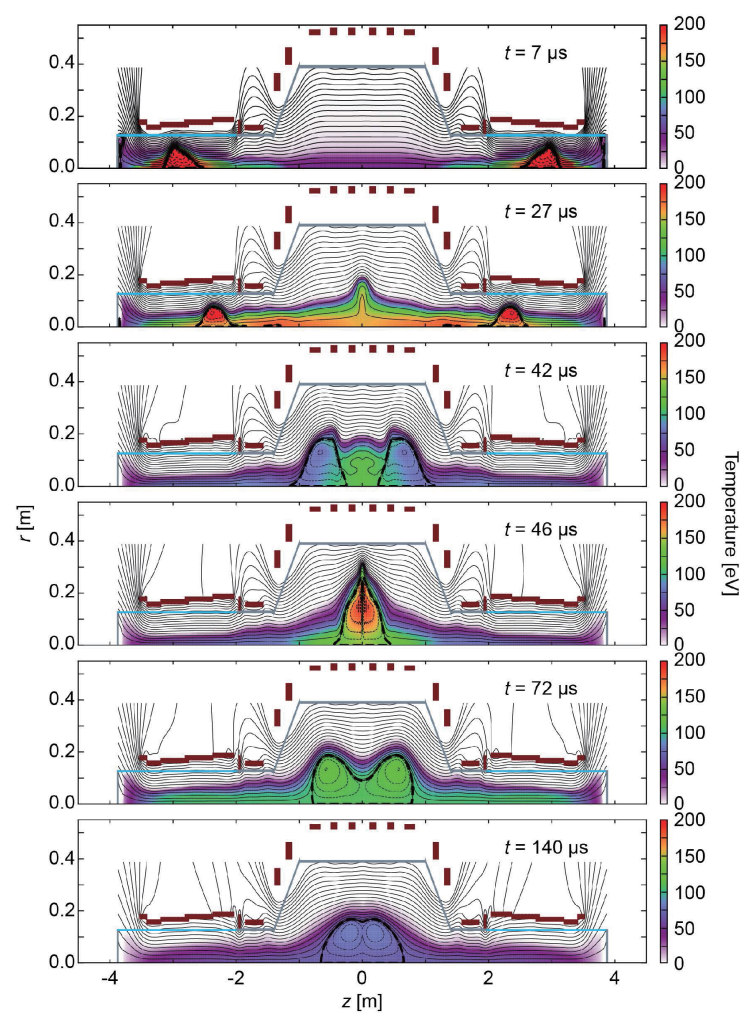

Fig. 2 Contour maps of plasma temperature computed by Lamy Ridge under the typical conditions of FAT-CM experiments.
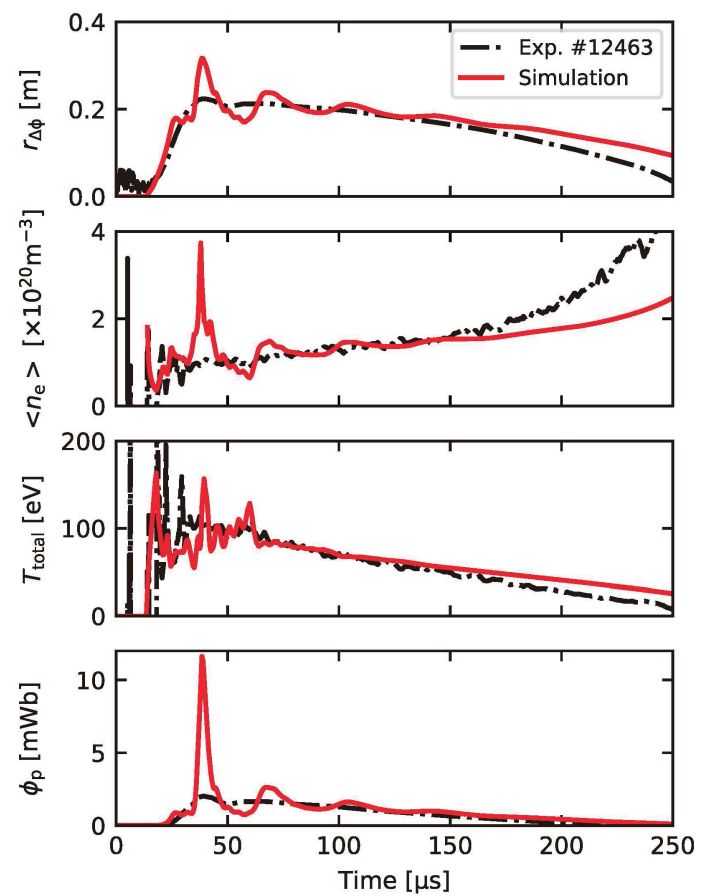

Fig. 3 Time evolution of excluded flux radius, averaged electron density, total temperature, and the trapped poloidal flux assuming the rigid-rotor equilibrium model [2].
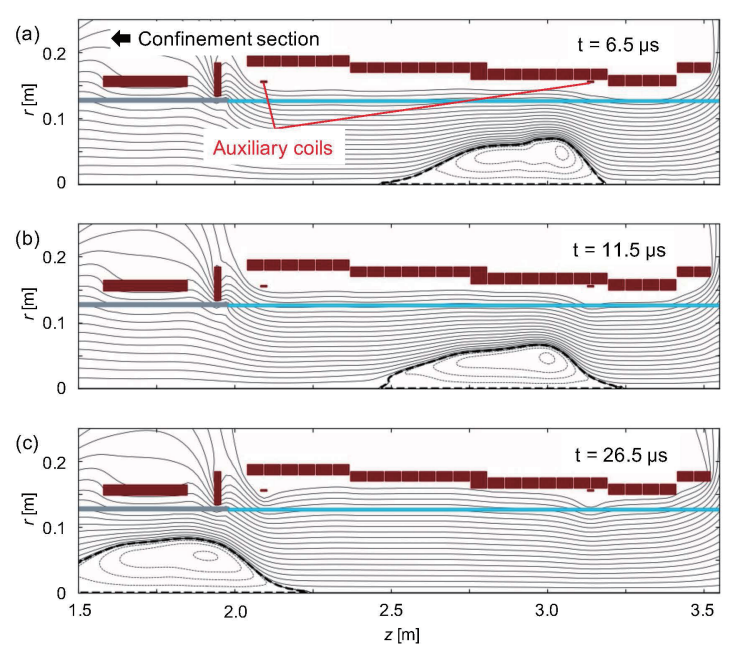

Fig. 4 Contour plot of the magnetic flux in "V-formation" during the acceleration of FRCs with auxiliary coils. Solid and dashed lines indicate positive and negative sections, respectively. The dashed bold line indicates the separatrix. (a) Shows the formed initial-FRC; (b) and (c) show FRC accelerated by an auxiliary coil.

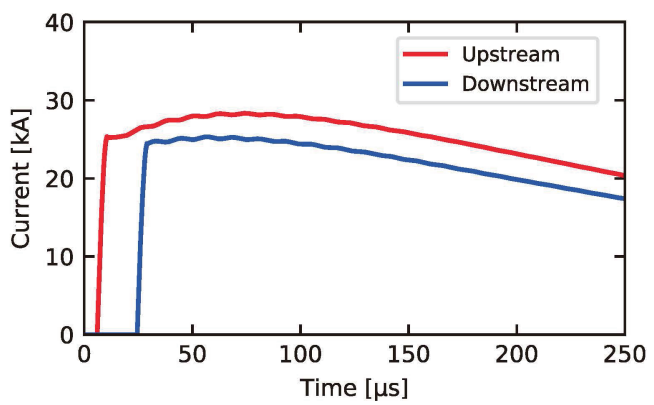

Fig. 5 Waveform of the current on auxiliary coils in the case of the maximum translation velocity presented in this paper (case 4).

\section{Auxiliary Coil}

The auxiliary coil was developed to control the translation of FRCs formed by the FRTP method [10]. In the previous study, a set of the auxiliary coils was used to separate an FRC into two parts; in addition, the ejection timing of each FRC was controlled by the gradient of the magnetic field changed by auxiliary coils.

The auxiliary coil is a set of one turn coils $(20 \mathrm{~mm}$ width, $3 \mathrm{~mm}$ thickness, $320 \mathrm{~mm}$ OD) that are installed on each formation. The contour plot of the magnetic flux function in "V-formation" during the acceleration of FRCs with auxiliary coils is shown in Fig. 4. Also, Fig. 5 shows the typical waveform of current on auxiliary coils in the simulation. These coils are used to change the profile of the external guide magnetic field. In the operation for acceleration, the current is triggered when FRCs are under the auxiliary coils, which increases the gradient of the external guide magnetic field (Figs. 4 (b), (c)). Then, FRCs are accelerated more than in the operation without auxiliary coils. 

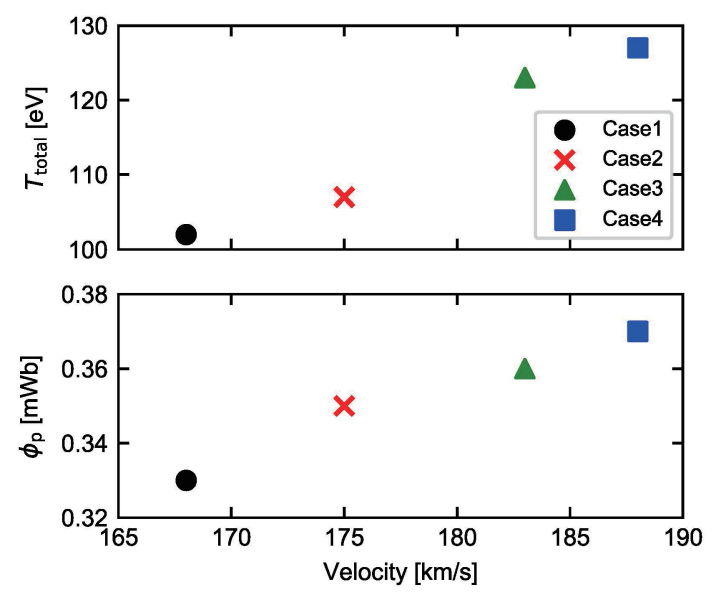

Fig. 6 Dependence of the total temperature and trapped poloidal flux on the maximum velocity during the translation.
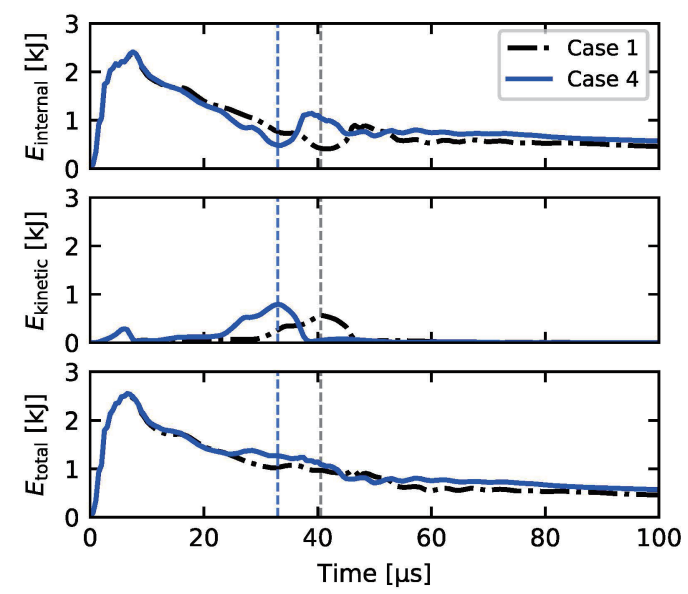

Fig. 7 Time evolution of internal, kinetic, and total energy of FRCs.

\section{Simulation Results}

Here, four cases are presented. In case 1, auxiliary coils are not used. In case 2, the coils on the downstream side, also in case 3 , and the coils on the upstream side are used. In case 4 , the coils on both sides are used.

The dependence of the total temperature, which is volume-averaged in separatrix, and the trapped poloidal flux at the time when the kinetic energy of FRCs became zero (i.e., after merging) on the translation velocity is shown in Fig. 6. Here, the average of the axial velocity of each fluid element in the separatrix is defined as the translation velocity of FRCs. FRCs have been accelerated using auxiliary coils in the simulation. In case 4 , the translation velocity is increased by up to $20 \mathrm{~km} / \mathrm{s}$. The translation velocity control by adjusting the number of installed coils, and their locations has been demonstrated. Furthermore, the averaged total temperature and the trapped poloidal flux increase depending on the translation velocity. Figure 7 shows the internal, kinetic, and total energy inside the separatrix in case 1 and case 4 . Translated FRCs collided at the time indicated by dashed lines in each case. The plots before the collision show the average energy of FRCs in each formation section. It is observed that kinetic energy is converted to internal energy in the collisional merging process. These results indicate that the total temperature and the trapped poloidal flux of merged-FRCs increase due to the higher kinetic energy of initial-FRCs.

\section{Summary}

Collisional merging formed FRC in the FAT-CM device has been reproduced in the simulation using the Lamy Ridge code. The performance of simulated FRCs was approximately consistent with that of experimental FRCs. These results indicate the usefulness of this code for evaluating the global behavior of FRCs during the collisional merging formation. The acceleration of FRCs with auxiliary coils has been evaluated by the simulations. The translation velocity control by adjusting the number of installed coils and their locations has been demonstrated on the order of several tens of $\mathrm{km} / \mathrm{s}$; in addition, the dependence of merged-FRC performance on translation velocity was observed in the simulation.

\section{Acknowledgement}

The authors would like to acknowledge all of members of Fusion-Plasma group, Nihon University and TAE who contributed to discussions. This work was partially supported by JSPS KAKENHI Grant Number JP19K21868, JP16K06939 and Grants-in-Aid of College of Science and Technology, Nihon University.

[1] M. Tuszewski, Nucl. Fusion 28, 2033 (1988).

[2] L.C. Steinhauer, Phys. Plasmas 18, 070501 (2011).

[3] S.V. Ryzhkov, Plasma Phys. Rep. 37, 1075 (2011).

[4] M.W. Binderbauer, H.Y. Guo, M. Tuszewski, S. Putvinski, L. Sevier et al., Phys. Rev. Lett. 105, 045003 (2010).

[5] T. Asai, Ts. Takahashi, J. Sekiguchi, D. Kobayashi, S. Okada et al., Nucl. Fusion 59, 056024 (2019).

[6] H. Guo, M. Binderbauer, D. Barnes, S. Putvinski, N. Rostoker et al., Phys. Plasmas 18, 056110 (2011).

[7] Y. Mok, D. Barnes and S. Dettrick, Bull. Am. Phys. Soc. 55, GP9.97 (2010).

[8] M. Onofri, P. Yushmanov, S. Dettrick, D. Barnes et al., Phys. Plasmas 24, 092518 (2017).

[9] F. Tanaka, T. Asai, J. Sekiguchi, Ts. Takahashi, J. Ishiwata et al., Plasma Fusion Res. 13, 3402098 (2018).

[10] Y. Matsuzawa, T. Asai, Ts. Takahashi and T. Takahashi, Phys. Plasmas 15, 082504 (2008). 